\title{
recivounds
}

Revista Científica Mundo de la Investígación y el Conocimiento

Myrian del Rocío Hidalgo Achig a; Marcela Patricia Vizuete Achig b; Mayra Alexandra Chicaiza Herrera ${ }^{c}$; Isabel Regina Armas Heredia ${ }^{\text {d}}$; Edison Patricio

Bedon Salazar ${ }^{\mathrm{e}}$; Patricia Geraldina López Fraga ${ }^{\mathrm{f}}$

Estudio de costos invertidos en la formación universitaria de los estudiantes de la

Facultad de Ciencias Administrativas de la Universidad Técnica de Cotopaxi

Study of costs invested in the university education of the students of the Faculty of Administrative Sciences of the Technical University of Cotopaxi

Revista Científica Mundo de la Investigación y el Conocimiento. Vol. 3 núm.2, abril, ISSN: 2588-073X, 2019, pp. 1130-1158

DOI: $10.26820 /$ recimundo/3.(2).abril.2019.1130-1158

URL: http://recimundo.com/index.php/es/article/view/494

Código UNESCO: 5303 Contabilidad Económica

Tipo de Investigación: Artículo de Investigación

Editorial Saberes del Conocimiento

Recibido: 15/01/2019

Aceptado: 18/02/2019

Publicado: 30/04/2019

Correspondencia: myrian.hidalgo@utc.edu.ec

a. Universidad Técnica de Cotopaxi; myrian.hidalgo@utc.edu.ec

b. Universidad Técnica de Cotopaxi; marcela.vizuete@utc.edu.ec

c. Universidad Técnica de Cotopaxi; mayra.chicaiza@utc.edu.ec

d. Universidad Técnica de Cotopaxi; isabel.armas@utc.edu.ec

e. Universidad Técnica de Cotopaxi; edison.bedon@utc.edu.ec

f. Universidad Técnica de Cotopaxi; patricia.lopez@utc.edu.ec 


\section{Estudio de costos invertidos en la formación universitaria de los estudiantes de la Facultad de Ciencias Administrativas de la Universidad Técnica de \\ Cotopaxi}

Vol. 3, núm. 2., (2019)

Myrian del Rocío Hidalgo Achig; Marcela Patricia Vizuete Achig; Mayra Alexandra Chicaiza Herrera; Isabel Regina Armas Heredia; Edison Patricio Bedon Salazar; Patricia Geraldina López

Fraga

\section{RESUMEN}

La razón de que las universidades sean instituciones cada día más visibles, producto de su evolución en las últimas décadas, hace necesario que estas tengan la necesidad de justificar la inversión de sus recursos en la formación universitaria. La razón de querer disponer de costos de las actividades universitarias se puede enfocar desde dos perspectivas, la primera contar con un instrumento de gestión interna que permita el control a los responsables de las diferentes actividades asignadas; $y$, como segundo aspecto como instrumento externo para producir indicadores que muestren a los grupos sociales interesados la productividad de los recursos que les han sido confiados, razón por la cual la presente investigación describe el estudio de los costos invertidos en la formación universitaria de los estudiantes de la Facultad de Ciencias Administrativas (FCA) de la Universidad Técnica de Cotopaxi (UTC). El objetivo es establecer los costos invertidos en la formación universitaria de los estudiantes de FCA de la UTC basado en los preceptos del sistema de costos ABC (Activity Based Costing) y el marco legal que rige a la Educación Superior. La metodología utilizada es de es de tipo documental y estudio de casos las cuales permitieron identificar la dinámica de las finanzas en la educación superior. Los resultados obtenidos permitieron identificar el modelo de Contabilidad Analítica para la Facultad de Ciencias Administrativas de la UTC, así como el reconocimiento de las unidades (centros) principales asociadas a la docencia, investigación y extensión; las de dirección y las auxiliares, concluyendo con la identificación de los costos por su naturaleza, actividades que se realizan en cada una de las carreras y la asignación de los costos respectivos, lo que permitió conocer el costo invertido en cada estudiante en las distintas carreras que se ofrecen la FCA de la UTC, lo que permite medir la gestión de la facultad entre la imaginación de los responsables académicos, imprescindible para el progreso de la universidad, y las limitaciones que supone la existencia de un presupuesto.

Palabras Claves: Costos; Educación Superior; Formación Universitaria; Costos ABC; Metodología. 


\section{Estudio de costos invertidos en la formación universitaria de los estudiantes de la Facultad de Ciencias Administrativas de la Universidad Técnica de Cotopaxi}

Vol. 3, núm. 2., (2019)

Myrian del Rocío Hidalgo Achig; Marcela Patricia Vizuete Achig; Mayra Alexandra Chicaiza Herrera; Isabel Regina Armas Heredia; Edison Patricio Bedon Salazar; Patricia Geraldina López Fraga

\section{ABSTRACT}

The reason why universities are increasingly visible institutions, a product of their evolution in recent decades, makes it necessary that they have the need to justify the investment of their resources in university education. The reason for wanting to have costs for university activities can be approached from two perspectives, the first having an internal management tool that allows control to those responsible for the different activities assigned; and, as a second aspect as an external instrument to produce indicators that show interested social groups the productivity of the resources entrusted to them, which is why this research describes the study of the costs invested in the university education of students of the Faculty of Administrative Sciences (FCA) of the Technical University of Cotopaxi (UTC). The objective is to establish the costs invested in the university education of the students of FCA of the UTC based on the precepts of the ABC cost system (Activity Based Costing) and the legal framework that governs Higher Education. The methodology used is of documentary type and case studies which allowed to identify the dynamics of finance in higher education. The results obtained allowed to identify the Analytical Accounting model for the Faculty of Administrative Sciences of the UTC, as well as the recognition of the main units (centers) associated with teaching, research and extension; management and auxiliary, concluding with the identification of costs by their nature, activities carried out in each of the races and the allocation of the respective costs, which allowed to know the cost invested in each student in the different races that the FCA of the UTC is offered, what allows to measure the management of the faculty between the imagination of the academic responsible, indispensable for the progress of the university, and the limitations that supposes the existence of a budget.

Key Words: Costs; Higher Education; University Education; ABC Costs; Methodology. 


\section{Estudio de costos invertidos en la formación universitaria de los estudiantes de la Facultad de Ciencias Administrativas de la Universidad Técnica de Cotopaxi}

Vol. 3, núm. 2., (2019)

Myrian del Rocío Hidalgo Achig; Marcela Patricia Vizuete Achig; Mayra Alexandra Chicaiza Herrera; Isabel Regina Armas Heredia; Edison Patricio Bedon Salazar; Patricia Geraldina López

Fraga

\section{Introducción.}

Actualmente, constituye una realidad que la gestión de los recursos públicos, en general, y los universitarios, en particular, demuestren un manejo eficiente de estos, , el reto de estas instituciones es el de optimizar recursos que provienen de financiamiento privado o del estado, es imperante y debe estar atado necesariamente a la posibilidad cierta de brindar una educación de excelencia para contribuir con el capital humano y el desarrollo del país, más aún en entornos donde la evaluación y la acreditación están cada día más en la palestra y determinan hasta la permanencia de la institución de educación superior.

El rol social que juegan las universidades en la actualidad "obliga, cada vez en mayor medida, a una aplicación eficiente y transparente de los recursos que la sociedad dedica a su cobertura" (López, 2011, p. 31), es por ello que en los últimos años se han desarrollado investigaciones vinculadas al diseño y aplicación de modelos de gestión colocando su énfasis en el estudio de los costos, ya sean los basados en actividades o ABC (Activity Based Costing) tal como plantean autores como Ficco (2011) o el modelo de contabilidad analítica propuesto por las Universidades públicas españolas.

El modelo de costos ABC como herramienta de gestión estratégica fue empleado en estudios como los de Ficco (2011) para analizar los costos, inicialmente en la Universidad Nacional de Río Cuarto y luego replicar en las Universidades Públicas de Argentina, pues esta metodología "permite no solo obtener información sobre costos de mejor calidad que otros 


\section{Estudio de costos invertidos en la formación universitaria de los estudiantes de la Facultad de Ciencias Administrativas de la Universidad Técnica de Cotopaxi}

Vol. 3, núm. 2., (2019)

Myrian del Rocío Hidalgo Achig; Marcela Patricia Vizuete Achig; Mayra Alexandra Chicaiza Herrera; Isabel Regina Armas Heredia; Edison Patricio Bedon Salazar; Patricia Geraldina López Fraga

sistemas de costeo, sino también, (...) por las posibilidades que brinda, como herramienta de gestión estratégica de esos costos" (p. 30).

Por su parte, la propuesta utilizada por las universidades públicas españolas como parte del proceso de reforma que ha experimentado el sistema educativo, se inicia en el año 1991 con el Sistema de Contabilidad Analítica para Universidades, que tres años más tarde da lugar al Proyecto CANOA (Contabilidad Analítica Normalizada de Organismos Autónomos), el cual “generó un destacado interés en el ámbito universitario, por adaptar el mismo a las características particulares de la universidad pública" (López, 2011, p. 34). Es en el año 2004 cuando se aprueba el sistema de contabilidad analítica y desde allí se emplea como marco normativo para la gestión de las universidades españolas, dando lugar en 2011 al denominado libro blanco de los costes para las universidades.

Este modelo de contabilidad analítica para las universidades no solo propone un sistema de costos, sino que incluye elementos de los ingresos e indicadores para gestionar estratégicamente los recursos asignados. Sin lugar a dudas, en este modelo el rol protagónico lo asume la información relacionada a los costos en la universidad, para lo cual se plantea la utilización de un sistema de costos por centros de costos basados en actividades.

De tal manera, se identifican los centros de costos que son las unidades o departamentos que consumen recursos, y además las actividades desarrolladas en la institución y en cada unidad, para luego imputar los costos a cada una. El objetivo final de la investigación fue el 


\section{Estudio de costos invertidos en la formación universitaria de los estudiantes de la Facultad de Ciencias Administrativas de la Universidad Técnica de \\ Cotopaxi}

Vol. 3, núm. 2., (2019)

Myrian del Rocío Hidalgo Achig; Marcela Patricia Vizuete Achig; Mayra Alexandra Chicaiza Herrera; Isabel Regina Armas Heredia; Edison Patricio Bedon Salazar; Patricia Geraldina López

Fraga

determinar el costo de cada actividad, del departamento o unidad, lo que proporciona una estimación cierta del costo de inversión en educación de cada estudiante y sus diferencias en cuanto a carreras si existieran.

El implementar estos sistemas representa un aporte importante a la gestión estratégica de las instituciones, es por ello, que la Universidad Técnica de Cotopaxi, dado que además de ser una universidad de gestión pública y por tanto debe responder por los aportes recibidos de la sociedad, orienta su gestión administrativa y financiera a la optimización en el uso de los recursos brindando una educación de excelencia.

En esta línea de acción la presente investigación tiene como propósito aplicar el modelo de contabilidad analítica para el período enero - diciembre de 2017 en la Universidad Técnica de Cotopaxi, respondiendo a interrogantes como ¿cuáles son los elementos de costos?, ¿cuáles son los departamentos o centros de costos en la FCA de la UTC? ¿Cuáles son las actividades desarrollas? ¿Cuáles son las carreras más costosas, existen diferencias en los costos por tipo de centro?

Para lo cual se inició con el desarrollo de una prueba piloto aplicada a la Facultad de Ciencias Administrativas que abarca las carreras de Contabilidad y Auditoría, Ingeniería Comercial y Secretariado Ejecutivo Gerencia.

\section{Marco Teórico}

Contabilidad Analítica 


\section{Estudio de costos invertidos en la formación universitaria de los estudiantes de la Facultad de Ciencias Administrativas de la Universidad Técnica de Cotopaxi}

Vol. 3, núm. 2., (2019)

Myrian del Rocío Hidalgo Achig; Marcela Patricia Vizuete Achig; Mayra Alexandra Chicaiza Herrera; Isabel Regina Armas Heredia; Edison Patricio Bedon Salazar; Patricia Geraldina López Fraga

La modernización del sector público insirió en su contexto innovaciones que contribuyeron con la necesidad de que los gestores públicos conozcan los costes parciales y totales de sus entidades, con la finalidad de tomar las mejores decisiones entre las alternativas de producir, comprar, subcontratar o privatizar

De acuerdo a (Martinez Navajas, 2006) señala que la contabilidad analítica se conoce también como:

“Contabilidad interna, de costes, de gestión, gerencial, directiva, etc. La contabilidad analítica está hecha para y por la empresa. La contabilidad analítica es principalmente un conjunto de técnicas que persigue la determinación analítica del resultado de la empresa. La contabilidad analítica es una rama de la contabilidad que analiza cómo se distribuyen los costes y los ingresos que genera una empresa. Trata de ver cuál es el coste de cada producto, de cada departamento, de cada cliente..., y ver que rentabilidad obtiene de cada uno de ellos". (p.3), con la finalidad de "ayudar en el proceso de toma de decisiones, a la elaboración de la planificación, a los sistemas de gestión del rendimiento, y a la prestación de habilidades relacionadas con la información financiera y de control, lo que ayuda a los administradores en la formulación y aplicación de la estrategia en la organización” (Lunkes, Ripoll, \& Silva, 2012, p.12)

De acuerdo a (Goncalves, Do Nascimento, Dos Santos, \& Bernardes, 2013) señalan que

Rezende, Cunha y Cardoso (2010), la importancia de las informaciones sobre los costes del 


\section{Estudio de costos invertidos en la formación universitaria de los estudiantes de la Facultad de Ciencias Administrativas de la Universidad Técnica de \\ Cotopaxi}

Vol. 3, núm. 2., (2019)

Myrian del Rocío Hidalgo Achig; Marcela Patricia Vizuete Achig; Mayra Alexandra Chicaiza Herrera; Isabel Regina Armas Heredia; Edison Patricio Bedon Salazar; Patricia Geraldina López

Fraga

gobierno no se resume a la contribución que ellas pueden dar para la eficiencia y la eficacia del gasto público, sino también por lo que representa a la luz de la necesidad de ser promovida una profunda reforma de la gestión pública en el país, indispensable para que el poder público pueda responder adecuadamente a los desafíos contemporáneos que se presentan a la sociedad” (p.176)

En definitiva, la contabilidad analítica trata como se distribuyen los costes y los ingresos generados u obtenidos por la institución, ayudando así a determinar la rentabilidad de cada departamento, sección y actividad, por lo que su objetivo se enmarca en medir el aprovechamiento de los recursos y comprobar si su uso está siendo eficiente.

Es así que se han desarrollado varios trabajos relacionados con la contabilidad de gestión, los cuales concuerdan que este tipo de contabilidad es útil para que la dirección de la empresa pueda tomar buenas decisiones administrativas. Esto se manifiesta desde los trabajos de Brown y Gardner (1985a), Brown, Gardner, y Vasarhelyi (1987), Shields (1997), hasta los de Pérez, Gallardo, y Peña (2005), Schekaiban y Ripoll (2005), Hesford et al. (2007), c. Con el fin de ubicar el trabajo investigativo dentro de la disciplina, (Lunkes, Ripoll, \& Silva, 2012) han clasificado la contabilidad de gestión en "tres grandes líneas de investigación y dentro de cada una de ellas en temas. Las tres líneas que hemos abiertos han sido: Línea 1. Costes. Línea 2. Planificación y Control y Línea 3. Otros temas dentro del contexto de la contabilidad de gestión. La línea de investigación Costes recoge los cuatro temas a continuación: Contabilidad de Costes, Gestión de costes (se analizan los cambios en el coste), estudios y aplicaciones prácticas de coste, y en último lugar Investigación en Costes.” (p. 14) 


\section{Estudio de costos invertidos en la formación universitaria de los estudiantes de la Facultad de Ciencias Administrativas de la Universidad Técnica de Cotopaxi}

Vol. 3, núm. 2., (2019)

Myrian del Rocío Hidalgo Achig; Marcela Patricia Vizuete Achig; Mayra Alexandra Chicaiza Herrera; Isabel Regina Armas Heredia; Edison Patricio Bedon Salazar; Patricia Geraldina López Fraga

Para el desarrollo de esta investigación se trabajó con el sistema ABC (Bravo Valdivieso, 2007) señala que el sistema de costos basado en actividades (ABC.) es una técnica de determinación de los costos que aparece a mediados de la década de los ochenta como una propuesta de aporte a las falencias de los sistemas de costos tradicionales, los promotores de este sistema son Robin Cooper y Roberto Kaplan quienes determinaron que el costo de los productos debe comprender el costo de las actividades necesarias para fabricarlo. Este sistema conocido por la denominación anglosajona "ActivityBasedCosting" - $\mathrm{ABC}$, se presenta como una herramienta útil para el análisis del costo y seguimiento de actividades, factores relevantes para el desarrollo y resultado final de la gestión empresarial para hacer frente a las decisiones coyunturales. Este sistema permite la asignación y distribución de los COSTOS INDIRECTOS de acuerdo a las actividades realizadas identificando el origen del costo de la actividad. El costo del producto o servicios se obtiene sumando los costos de las actividades que intervienen en el proceso. (p.307)

De esta manera el sistema de costos $\mathrm{ABC}$ es un modelo gerencial y no un modelo contable, los recursos son consumidos por las actividades y éstas a su vez por los objetos de costos, considera todos los costos y gastos como recursos, muestra la empresa como un conjunto de actividades y/o procesos más que una jerarquía departamental, es una metodología que asigna costos a los productos o servicios con base en el consumo de actividades. El valor agregado de la aplicación del sistema $\mathrm{ABC}$ se resume en los resultados que obtiene la organización, tale s 


\section{Estudio de costos invertidos en la formación universitaria de los estudiantes de la Facultad de Ciencias Administrativas de la Universidad Técnica de \\ Cotopaxi}

Vol. 3, núm. 2., (2019)

Myrian del Rocío Hidalgo Achig; Marcela Patricia Vizuete Achig; Mayra Alexandra Chicaiza Herrera; Isabel Regina Armas Heredia; Edison Patricio Bedon Salazar; Patricia Geraldina López

Fraga

como: reducción de los ciclos de tiempo, reducción de costos y consecuente mejoramiento de la calidad y el incremento de la productividad. (p.308)

Para (Zapata, 2007) se señala que para definir la Contabilidad de Costos es necesario conocer plenamente qué, cómo, cuándo, porqué, para qué, y quién usa ese objeto. Además, manifiesta que las bases técnicas aplicables a la Contabilidad de Costos se rigen por las normas nacionales, internacionales y conceptos generalmente aceptados que regulan los procedimientos de trabajo a fin de hacerlos uniformes y confiables. (p.19)

\section{Estructura de los Costos}

Se entiende por "coste" la medida y valoración del consumo realizado o previsto por la aplicación racional de los factores para la obtención de un producto, trabajo o servicio. (Macias, 2011). Por lo expuesto se puede indicar que las instituciones deben identificar el sistema de costos adecuado a sus actividades y necesidades de información.

La propuesta metodológica se enfocó a establecer los costos invertidos en la formación universitaria de los estudiantes de FCA de la UTC basado en los preceptos del sistema de costos $\mathrm{ABC}$ (Activity Based Costing) y el marco legal que rige a la Educación Superior.

En base a lo señalado los costos incurridos en la actividad de educación superior son:

- Docencia.

- Investigación. 


\section{Estudio de costos invertidos en la formación universitaria de los estudiantes de la Facultad de Ciencias Administrativas de la Universidad Técnica de Cotopaxi}

Vol. 3, núm. 2., (2019)

Myrian del Rocío Hidalgo Achig; Marcela Patricia Vizuete Achig; Mayra Alexandra Chicaiza Herrera; Isabel Regina Armas Heredia; Edison Patricio Bedon Salazar; Patricia Geraldina López Fraga

- Gestión, y.

- Vinculación.

De acuerdo a lo señalado por la LOES establece en su Art. 24.- Distribución de los recursos.- Los recursos destinados anualmente por parte del Estado a favor de las universidades, escuelas politécnicas, institutos superiores técnicos, tecnológicos, pedagógicos, de artes y los conservatorios superiores públicos y particulares que reciban rentas y asignaciones del Estado, se distribuirán con base a criterios de calidad, eficiencia, equidad, justicia y excelencia académica, que entre otros parámetros prevalecerán los siguientes:

- Número de estudiantes y costo por carrera y nivel;

- Número, dedicación, título y experiencia docente en función de las evaluaciones pertinentes;

- Clasificación académica y tipología de instituciones, carreras y programas;

- Eficiencia en docencia e investigación y relación con el desarrollo nacional y regional;

- Eficiencia terminal; y Administrativa.

Para la propuesta se consideró como base de asignación el número de estudiantes de las carreras que forman parte de la Facultad de Ciencias Administrativas, lo cual evidenciara la estructura de los costos del funcionamiento institucional en su esencia educativa y que se traduce en la producción de su producto esencial como los estudiantes egresados y graduados. 


\section{Estudio de costos invertidos en la formación universitaria de los estudiantes de la Facultad de Ciencias Administrativas de la Universidad Técnica de Cotopaxi}

Vol. 3, núm. 2., (2019)

Myrian del Rocío Hidalgo Achig; Marcela Patricia Vizuete Achig; Mayra Alexandra Chicaiza Herrera; Isabel Regina Armas Heredia; Edison Patricio Bedon Salazar; Patricia Geraldina López

Fraga

\section{Metodología.}

La Universidad Ecuatoriana está obligada a desarrollar herramientas que le permitan el procesamiento y trasmisión de la información para los distintos usuarios interesados, desde los gestores de dichas instituciones a los distintos niveles de decisión, hasta los estamentos políticos responsables de la asignación de fondos para su financiación, o la propia sociedad que debe valorar la acción de las mismas (futuros alumnos, empresas, etc.), participar y beneficiarse de los resultados formativos y de generación de nuevos conocimientos que caracterizan la actividad de la Universidad

El desarrollo de proyecto inicia con una recopilación de información de fuentes bibliográficas primarias y secundarias que permitieron identificar la dinámica de los costos en las instituciones de Educación Superior, principalmente en la Universidad Técnica de Cotopaxi en la Facultad de Ciencias Administrativas para posteriormente determinar su asignación y acumulación a las distintas carreras.

Para el análisis se hizo referencia al marco legal que regula la las Instituciones de Educación Superior del país (IES) partiendo de la revisión de la Constitución de la república, leyes, reglamentos, disposiciones emanadas por organismos de control así; así también, la normativa institucional es merecedora de un análisis exhaustivo para lograr entender la interrelación de la ejecución presupuestaria en función de las actividades sustantivas de la UTC. 


\section{Estudio de costos invertidos en la formación universitaria de los estudiantes de la Facultad de Ciencias Administrativas de la Universidad Técnica de Cotopaxi}

Vol. 3, núm. 2., (2019)

Myrian del Rocío Hidalgo Achig; Marcela Patricia Vizuete Achig; Mayra Alexandra Chicaiza Herrera; Isabel Regina Armas Heredia; Edison Patricio Bedon Salazar; Patricia Geraldina López Fraga

\section{Cuadro No. 1 Marco Legal y normativo}

\begin{tabular}{|c|c|}
\hline LEY, REGLAMENTO & ARTICULOS REFERENCIA \\
\hline \multirow{3}{*}{ Constitución de la República del Ecuador } & Art. 3 Deberes primordiales del Estado \\
\hline & Art. 26, 27, 28, 29 Derechos del Buen Vivir \\
\hline & Art. $343,344,345,346,347,348$ y 349 Régimen del Buen Vivir \\
\hline \multirow{2}{*}{ Ley Orgánica de Educación Superior } & Art.3 Fines de la Educación Superior \\
\hline & Art. 4 Derecho a las Educación Superior \\
\hline \multicolumn{2}{|c|}{ Reglamento de la Ley Orgánica de Educación Superior } \\
\hline \multicolumn{2}{|l|}{ Reglamento del Régimen Académico } \\
\hline \multicolumn{2}{|l|}{ Estatutos de la Universidad Técnica de Cotopaxi } \\
\hline \multicolumn{2}{|l|}{ Reglamentos de Investigación, vinculación } \\
\hline \multicolumn{2}{|l|}{ Reglamento del Régimen Académico de la UTC } \\
\hline Plan Estrategico de la UTC & \\
\hline
\end{tabular}

Fuente: Revisión bibliográfica de disposiciones legales referidas para el levantamiento de información para el desarrollo de la metodología para el análisis de los costos en la universidad.

Para efectos del análisis de los costos se estructura el siguiente flujograma que permite el reconocimiento de los costos y su posterior análisis hasta lograr obtener informes de cada carrera de la Facultad de Ciencias Administrativas de la UTC: 


\section{Estudio de costos invertidos en la formación universitaria de los estudiantes de la Facultad de Ciencias Administrativas de la Universidad Técnica de Cotopaxi}

Vol. 3, núm. 2., (2019)

Myrian del Rocío Hidalgo Achig; Marcela Patricia Vizuete Achig; Mayra Alexandra Chicaiza Herrera; Isabel Regina Armas Heredia; Edison Patricio Bedon Salazar; Patricia Geraldina López

Fraga

\section{Figura No. 1 Metodología para determinación de costos por carreras de la Facultad de}

\section{Ciencias Administrativas de la UTC}

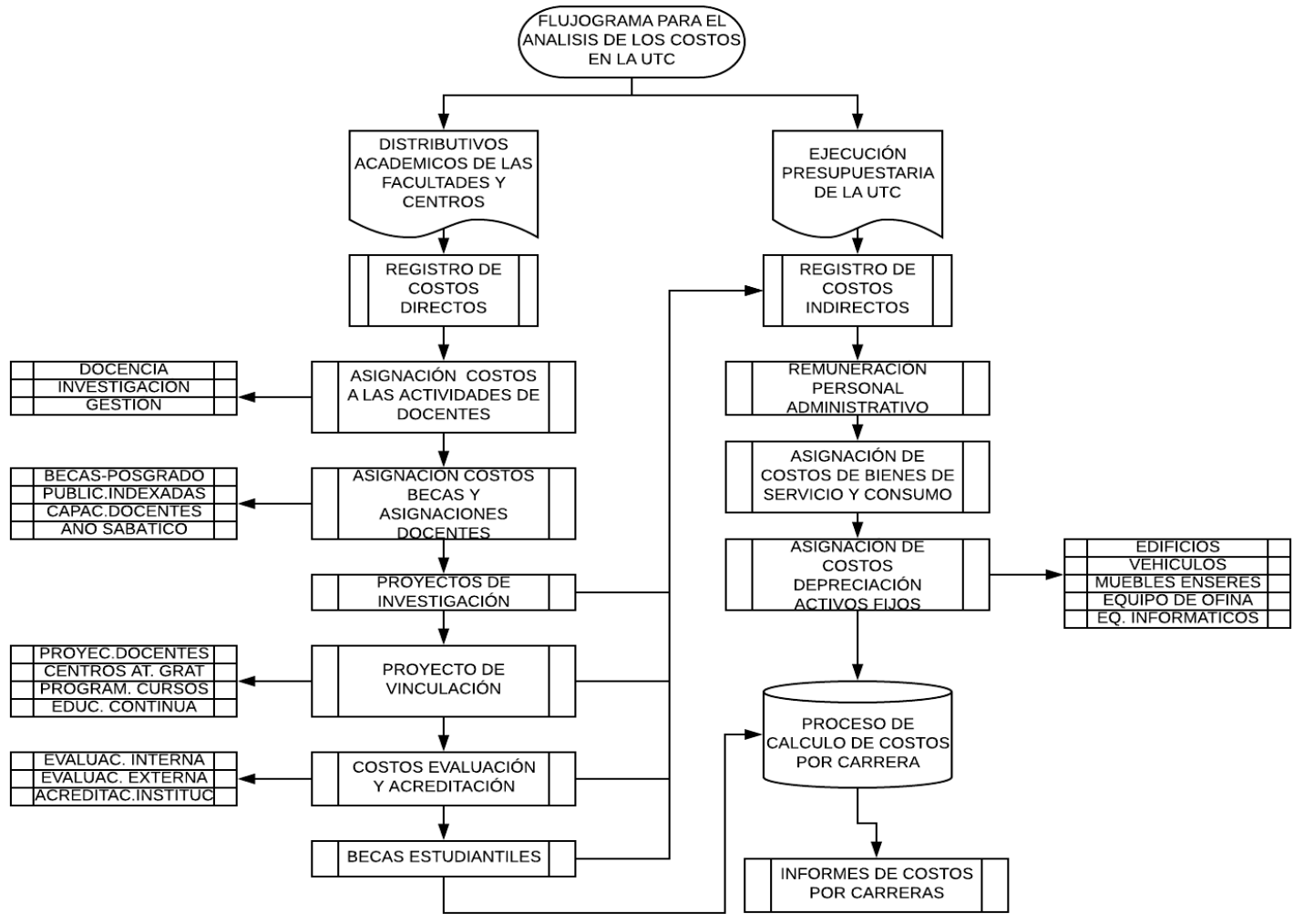

En la figura No. 1 se describe el flujograma de actividades para ejecutar el análisis de costos de la Facultad de Ciencias Administrativas de la UTC, se distingue los costos directos e indirectos y el proceso que se debe seguir hasta procesar la información y obtener informes de los costos por cada carrera de la facultad. 


\section{Estudio de costos invertidos en la formación universitaria de los estudiantes de la Facultad de Ciencias Administrativas de la Universidad Técnica de Cotopaxi}

Vol. 3, núm. 2., (2019)

Myrian del Rocío Hidalgo Achig; Marcela Patricia Vizuete Achig; Mayra Alexandra Chicaiza Herrera; Isabel Regina Armas Heredia; Edison Patricio Bedon Salazar; Patricia Geraldina López Fraga

Para efecto de dar cumplimiento este flujograma se describe los criterios técnicos que se toma en cuenta para lograr una eficaz distribución de los costos de acuerdo a la actividad. Como primera fase del proyecto se tomó como pilotaje a la Facultad de Ciencias Administrativas, con sus tres carreras: Comercio, Contabilidad y Auditoría y Secretariado Ejecutivo Gerencial; de docentes tanto en el campus Matriz y la Extensión La Mana en el caso de las carreras de Comercio y Contabilidad

Como segundo fase fue necesario identificar costos directos e indirectos ejecutados por la institución, información proporcionada por la dirección financiera, costos que fueron distribuidos a las diferentes carreras considerando el número de estudiantes de cada una de ellas en periodo a investigar.

Se considera como COSTOS DIRECTOS aquellos que se encuentran relacionados directamente con las actividades principales que aportan al cometido principal de la universidad; actividades como:

- La docencia,

- La investigación,

- La gestión y

- Actividades de vinculación con la comunidad (las actividades de vinculación se encuentran inmersas en las actividades de docencia e investigación) 


\section{Estudio de costos invertidos en la formación universitaria de los estudiantes de la Facultad de Ciencias Administrativas de la Universidad Técnica de \\ Cotopaxi}

Vol. 3, núm. 2., (2019)

Myrian del Rocío Hidalgo Achig; Marcela Patricia Vizuete Achig; Mayra Alexandra Chicaiza Herrera; Isabel Regina Armas Heredia; Edison Patricio Bedon Salazar; Patricia Geraldina López

Fraga

- Partidas presupuestarias establecidas por la institución destinada a financiar planes de becas o ayudas económicas para especialización o capacitación y año sabático. La Universidad Técnica de Cotopaxi dispone dentro de su presupuesto una partida destinada a los siguientes rubros:

\section{Becas Docentes}

2. Ayudas económicas para especialización o capacitación

3. Año sabático.

- Otra partida que se consideró fue la destinada a ejecutar proyectos de investigación, adquirir infraestructura tecnológica, publicar en revistas indexadas de alto impacto, otorgar becas doctorales a sus profesores titulares y pago de patentes.

- Asignación de los costos de evaluación y acreditación

- Costos de becas estudiantiles, la institución establece programas de becas completas o su equivalente en ayudas económicas que apoyen la escolaridad a por lo menos el $10 \%$ del número de estudiantes regulares.

- Cálculo y asignación de los costos de biblioteca. 


\section{Estudio de costos invertidos en la formación universitaria de los estudiantes de la Facultad de Ciencias Administrativas de la Universidad Técnica de Cotopaxi}

Vol. 3, núm. 2., (2019)

Myrian del Rocío Hidalgo Achig; Marcela Patricia Vizuete Achig; Mayra Alexandra Chicaiza Herrera; Isabel Regina Armas Heredia; Edison Patricio Bedon Salazar; Patricia Geraldina López Fraga

\section{Distribución de Costos Directos}

Para el cálculo de los costos directos se inició con el análisis de distributivos, para identificar la asignación de trabajo del docente en actividades de docencia, investigación y gestión; para efectos de la presente investigación se describe de forma detallada el cálculo:

Análisis de distributivos de trabajo de docentes

Iniciando con el trabajo se realizó el análisis de distributivos docentes; considerando que en el año 2017 se despliega 3 diferentes distributivos de acuerdo a los siguientes periodos académicos:

- $\quad$ Octubre 2016 - febrero 2017.

- Abril - agosto 2017.

- Octubre 2017 - febrero 2018.

En el caso de los periodos octubre 2016 - febrero 2017 y octubre 2017 - febrero 2018 se contabiliza la distribución de trabajo tan solo de los meses de enero hasta diciembre del año que se analiza, el 2017, permitiendo conocer el número de horas laborados en el periodo.

Es importante indicar que el análisis se realiza conforme la Facultad y carrera al que pertenece el docente, tomando en consideración la asignación de trabajo ya sea en los campus Matriz, Salache y la extensión La Mana. 


\section{Estudio de costos invertidos en la formación universitaria de los estudiantes de la Facultad de Ciencias Administrativas de la Universidad Técnica de Cotopaxi}

Vol. 3, núm. 2., (2019)

Myrian del Rocío Hidalgo Achig; Marcela Patricia Vizuete Achig; Mayra Alexandra Chicaiza Herrera; Isabel Regina Armas Heredia; Edison Patricio Bedon Salazar; Patricia Geraldina López

Fraga

A modo de ejemplo se presenta la tabla No. 1, en la que se describe el formato de la matriz utilizada para el análisis de la distribución docente; se considera los periodos académicos relacionados al año 2017, así también se toma en cuenta los cambios de carrera y las horas asignadas de acuerdo a la asignación en actividades de docencia, investigación y gestión.

Docente: NN

\begin{tabular}{|c|c|c|c|c|c|c|c|c|}
\hline \multirow[b]{2}{*}{ ACTIVIDAD } & \multicolumn{2}{|c|}{ 2016-2017 } & \multicolumn{2}{|c|}{$2017-2017$} & \multicolumn{2}{|c|}{$2017-2018$} & \multicolumn{2}{|c|}{ RESUMEN } \\
\hline & HORAS & $\begin{array}{l}\text { TOTAL HORAS } \\
\text { LABORADAS }\end{array}$ & HORAS & $\begin{array}{l}\text { TOTAL HORAS } \\
\text { LABORADAS }\end{array}$ & HORAS & $\begin{array}{l}\text { TOTAL HORAS } \\
\text { LABORADAS }\end{array}$ & $\begin{array}{l}\text { TOTAL HORAS } \\
\text { ANUALES }\end{array}$ & INDICADOR \\
\hline \multicolumn{9}{|c|}{ CONTABILIDAD Y AUDITORIA } \\
\hline \multicolumn{9}{|l|}{ DOCENCIA } \\
\hline CONTABILIDAD Y AUDITORIA & 23 & 299 & 25 & 600 & & & 899 & $47 \%$ \\
\hline INVESTIGACIÓN & & 0 & & 0 & & & 0 & $0 \%$ \\
\hline PUBLICACIONES & 17 & 221 & 5 & 120 & & & 341 & $18 \%$ \\
\hline GESTIÓN & & 0 & & 0 & & & 0 & $0 \%$ \\
\hline CREACIÓN DE NUEVAS CARRERAS & & & 10 & 240 & & & 240 & $13 \%$ \\
\hline \multicolumn{7}{|c|}{ SECRETARIADO EJECUTIVO } & 0 & $0 \%$ \\
\hline DOCENCIA & & & & & & & 0 & $0 \%$ \\
\hline SECRETARIADO EJECUTIVO & & & & & 16 & 176 & 176 & $9 \%$ \\
\hline CENTRO DE IDIOMAS & & & & & 8 & 88 & 88 & $5 \%$ \\
\hline INVESTIGACIÓN & & & & & & 0 & 0 & $0 \%$ \\
\hline PRODUCCIÓN CIENTIFICA & & & & & 10 & 110 & 110 & $6 \%$ \\
\hline $\begin{array}{l}\text { PROYECTOS DE INVESTIGACION FORTALECIMIENTO DE LA ECONOMIA } \\
\text { POPULAR Y SOLIDARIA }\end{array}$ & & & & & 6 & 66 & 66 & $3 \%$ \\
\hline GESTIÓN & & & & & & 0 & 0 & $0 \%$ \\
\hline TOTAL & 40 & 520 & 40 & 960 & 40 & 440 & 1920 & $100 \%$ \\
\hline
\end{tabular}

Cuadro 2. Matriz de cálculo de horas docente laboradas en el año 2017

Este proceso es repetitivo y personalizado de acuerdo a la realidad de la institución y la distribución de cada docente de la Facultad y la carrera, obteniendo el número de horas anual y se propone una distribución proporcional basada en el peso de la actividad que tiene acorde a su distribución. 


\section{Estudio de costos invertidos en la formación universitaria de los estudiantes de la Facultad de Ciencias Administrativas de la Universidad Técnica de Cotopaxi}

Vol. 3, núm. 2., (2019)

Myrian del Rocío Hidalgo Achig; Marcela Patricia Vizuete Achig; Mayra Alexandra Chicaiza Herrera; Isabel Regina Armas Heredia; Edison Patricio Bedon Salazar; Patricia Geraldina López Fraga

Realizado este proceso de análisis, se procede al cálculo de los sueldos y beneficios sociales a los que se tiene derecho como docente universitario, en esta fase se consideró particularidades como: salida de docentes, ingreso de nuevos docentes y docentes permanentes a fin de calcular la proporción de beneficios sociales que se asigna en estos casos.

Para la ejecución de este proyecto se obtiene la siguiente matriz a fin de mostrar el análisis pormenorizado que se realizó por cada docente, carrera y facultad.

\begin{tabular}{|c|c|c|c|c|c|c|c|c|c|c|c|c|c|c|c|c|}
\hline № & CÉDULA & DOCENTE & FACULTAD & CARRERA & $\begin{array}{c}\text { TIPO DE } \\
\text { RELACIÓN }\end{array}$ & $\begin{array}{l}\text { SUELDO } \\
\text { NOMINAL } \\
\text { MENSUAL }\end{array}$ & $\begin{array}{l}\text { SUELDO } \\
\text { ANUAL }\end{array}$ & \begin{tabular}{|c|} 
DÉCIMO \\
TERCERO
\end{tabular} & \begin{tabular}{|l|} 
DÉCIMOO \\
CUARTO
\end{tabular} & \begin{tabular}{|c|} 
FONDOS \\
DE \\
RESERVA
\end{tabular} & $\begin{array}{l}\text { VACACION } \\
\text { ES } \\
\text { PAGADAS }\end{array}$ & \begin{tabular}{|c|} 
IESS \\
PATRONAL \\
\end{tabular} & $\begin{array}{c}\text { SUBROGACIO } \\
\text { NES } \\
\text { IENCARGOS }\end{array}$ & \begin{tabular}{|c|}
13 ERO \\
MENSUALIZAD \\
0 \\
\end{tabular} & \begin{tabular}{|c|} 
APORTE \\
PATRONAL \\
\end{tabular} & \begin{tabular}{|c|} 
TOTAL \\
INGRES \\
OS \\
\end{tabular} \\
\hline 1 & 1719715375 & ALBARRASIN REINOSO MARILIN VANESSA & CIENCIAS ADMNISTRATINAS & COMERCIO & NOMBRAMENTO & & 27600,00 & 2300,00 & 375,00 & 2299,08 & 2300,00 & 2525,40 & & & & 37399,48 \\
\hline & 0501655575 & ALTAMRANO BAUTISTA SLVVA HORTENCIA & CIENCIAS ADMNISTRATNAS & COMERCIO & CONTRATO & & 16290,00 & 1357,50 & 281,25 & 1356,96 & 1810,00 & 1490,54 & & & & 22586,24 \\
\hline 3 & 0502123730 & ARIAS FIGUEROA ROBERTO CARLOS & CIENCIAS ADMNISTRATINAS & COMERCIO & CONTRATO & & 27404,00 & 2283,67 & 375,00 & 2282,75 & 2300,00 & 2507,47 & & 10,25 & & 37152,89 \\
\hline 4 & 0501315857 & ALMACHE VACANESTOR EMLLO & CIENCIAS ADMNISTRATINAS & COMERCIO & CONTRATO & & 18142,70 & 1511,89 & 250,00 & 1511,29 & 1810,00 & 1660,06 & & & & 24885,94 \\
\hline 5 & 0502786833 & BORJA BRAZALES YADIRA PAOLA & CIENCIAS ADMNISTRATINAS & COMERCIO & CONTRATO & & 19910,00 & 1659,17 & 375,00 & 1658,50 & 1810,00 & 1821,77 & & & & 27234,43 \\
\hline 6 & 1803017753 & CRIOLLO SALNAS JENNY MARICELA & CIENCIAS ADMNISTRATNAS & COMERCIO & CONTRATO & & 9050,00 & 754,17 & 156,25 & 753,87 & 1810,00 & 828,08 & & & & 13352,36 \\
\hline
\end{tabular}

\section{Cuadro 3. Matriz de análisis de Sueldos y Beneficios Sociales Docente - FCA}

El cuadro No. 3 muestra el análisis de sueldos y beneficios sociales para obtener los ingresos anuales del docente y proceder al cálculo del rubro correspondiente a cada actividad; sin embargo al ser las dos matrices descritas anteriores auxiliares para el procesamiento de datos en un sistema automatizado, el trabajo actual se encuentra en el procesamiento e ingreso de datos en el sistema.

La idea del sistema se cristaliza con la intensión de evitar errores e inexactitudes en los cálculos para lograr costos reales que sirvan a quienes corresponda para la toma de decisiones. 


\section{Estudio de costos invertidos en la formación universitaria de los estudiantes de la Facultad de Ciencias Administrativas de la Universidad Técnica de \\ Cotopaxi}

Vol. 3, núm. 2., (2019)

Myrian del Rocío Hidalgo Achig; Marcela Patricia Vizuete Achig; Mayra Alexandra Chicaiza Herrera; Isabel Regina Armas Heredia; Edison Patricio Bedon Salazar; Patricia Geraldina López

Fraga

Posteriormente se identificaron los COSTOS INDIRECTOS que corresponden a aquellos gastos que tienen relación con las remuneraciones del personal administrativo que conforman la estructura orgánica diferente del personal Docente, depreciaciones de activos fijos, gastos por servicios, de bienes de consumo corriente, gastos de servicios informáticos. En cuanto al proceso para la distribución de los costos indirectos se determinó de la siguiente información:

1. Revisión de Ejecución Presupuestaria del año 2017.

2. Revisión de Balance de Comprobación del año 2017.

3. Revisión de matrices de pagos de servicios de básicos.

4. Depuración de cuentas sin partida presupuestaria.

5. Elaboración de la nomenclatura en relación a costos indirectos.

6. Constatación de medidores, aulas, laboratorios, oficinas, biblioteca, salas.

7. Revisión de mayores para determinar los diferentes movimientos de las cuentas que participan en los costos indirectos.

8. Elaboración de matriz con los respectivos indicadores relacionados al consumo y abastecimiento. 


\section{Estudio de costos invertidos en la formación universitaria de los estudiantes de la Facultad de Ciencias Administrativas de la Universidad Técnica de Cotopaxi}

Vol. 3, núm. 2., (2019)

Myrian del Rocío Hidalgo Achig; Marcela Patricia Vizuete Achig; Mayra Alexandra Chicaiza Herrera; Isabel Regina Armas Heredia; Edison Patricio Bedon Salazar; Patricia Geraldina López Fraga

La distribución de los costos indirectos se aplicó a las diferentes Facultades de la Universidad: Humanas, Ciencias Agropecuarias y Recursos Naturales, Administrativas y Aplicadas; Centros: Idiomas, Posgrado, Educación Continua y demás Dependencias Administrativas.

Para la ejecución del análisis y distribución de costos a las diferentes facultades y centros que consumen los costos se atribuye consideraron criterios para la distribución equitativa de los costos indirectos a cada centro y facultad de la universidad, estos criterios se consideraron de acuerdo a la realidad de la universidad y la ejecución del presupuesto.

En esta etapa, se realizaron las siguientes actividades:

1. Impresión de los mayores, en relación a los costos indirectos.

2. Visita al Departamento Financiero para solicitar información referente a los costos indirectos consumidos en las diferentes actividades que conforman la Institución.

3. Distribución de los costos indirectos considerando las Facultades: Humanas, Ciencias Agropecuarias y Recursos Naturales, Administrativas y Aplicadas; Centros: Idiomas, Posgrado, Educación Continua y demás Dependencias Administrativas.

4. Consulta a la Sra. Analista de Talento Humano sobre los Roles de Pago del Personal Administrativo y de Servicio. 


\section{Estudio de costos invertidos en la formación universitaria de los estudiantes de la Facultad de Ciencias Administrativas de la Universidad Técnica de Cotopaxi}

Vol. 3, núm. 2., (2019)

Myrian del Rocío Hidalgo Achig; Marcela Patricia Vizuete Achig; Mayra Alexandra Chicaiza Herrera; Isabel Regina Armas Heredia; Edison Patricio Bedon Salazar; Patricia Geraldina López

Fraga

5. Pedido a la Jefe de Servicios Generales la distribución del personal de: Servicio y Seguridad para proceder a la distribución de los Costos correspondientes.

En este proceso se generó matrices en las cuales se puede visualizar la distribución de los costos de acuerdo al rubro, estas matrices reflejan el trabajo realizado y el análisis del grupo de investigación, para mejor visualización se anexa al documento.

\section{Cuadro 4. Matriz de distribución de costos}

\begin{tabular}{|c|c|c|c|c|c|c|c|c|c|c|c|c|c|}
\hline rews | & с6́160 & inORERTO & $\begin{array}{c}\text { CoSTO/ NOOHEBBBE } \\
\text { MARR }\end{array}$ & GESTION & CCAA & 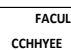 & ${ }_{\text {cra }}$ & CAREN & La Maná & 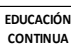 & IDIOMAS & POSGRADO & Dosservaciones \\
\hline 1 & 53010105001001 & Agua Potable & 3.4689 & & $\frac{1 C A, 5}{58,5}$ & & & & & & & & \\
\hline $\begin{array}{l}2 \\
3\end{array}$ & | 33010400501001 & Enegria Electrica & & & & & & & & & & & \\
\hline 4 & $\begin{array}{l}5301006501001 \\
501\end{array}$ & \begin{tabular}{|l} 
Telecomunicaciones \\
Servicio de Correo
\end{tabular} & 13392,19 & 38.50 & & & & & & & & & \\
\hline 5 & 53020405001002 & 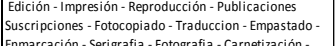 & 37954,08 & 35734,58 & & 239,90 & 364,00 & & 1615,6 & & & & \\
\hline & & 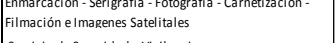 & & & & & & & & & & & \\
\hline 6 & 5302080 5050 001 & 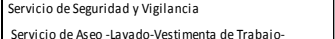 & 20567,74 & 1873,78 & & & & 18693,96 & & & & & \\
\hline 7 & 53020905001001 & 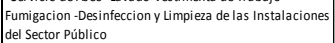 & 2004,16 & 220,17 & & & & 268399 & & & & & \\
\hline 8 & 53030010501002 & \begin{tabular}{|l|l} 
Passies a a Interior \\
\end{tabular} & 978,58 & 323,92 & & 313,79 & & & & & 340,87 & & \\
\hline 9 & & Passjes a le exterior & 31087,03 & 12373,93 & 5189,51 & 1895,05 & 5238,44 & 345,76 & 1162,94 & & 894 & 876 & \\
\hline 10 & 53030000501001 & Viaticos y yubsis itencias en el Interior & 14852,17 & 14153,03 & 80,00 & 240,00 & & 260,00 & & & 119,14 & & \\
\hline 11 & & Viaticos por gastos de resididencia & 7788,00 & 7788,00 & & & & & & & & & \\
\hline 12 & & 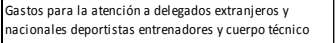 & 198,36 & & & & & & 198,36 & & & & \\
\hline 13 & 53030405001001 & 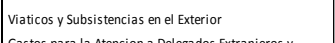 & 17224,89 & 15794,04 & & & & 705,60 & 725,25 & & & & \\
\hline 14 & 5303070501001 & 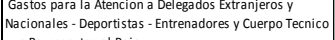 & 198,36 & & & & & & & & & & \\
\hline 15 & 5304040501002 & 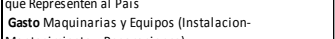 & 831244 & 604734 & & & & 2265.1 & & & & & \\
\hline 16 & 5304220501001 & 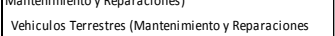 & 5981,23 & 5981,23 & & & & & & & & & \\
\hline 17 & 5300606501001 & Honorarrios por Contratasos Civiles de Servicios & 16488,73 & 4623,99 & 14497,3086 & 26030,07 & 38648,91 & 23971,17 & 539,06 & 8873,3 & 4222,06 & 2150 & \\
\hline 18 & 5306120501001 & Capacitación S Servidores Públicos & 96685,14 & 30800,63 & 18548,13 & 25667,19 & 18908,11 & 2651,08 & & & 110 & & \\
\hline 19 & 53070205501002 & 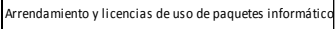 & ${ }_{15917,83}$ & 1344 & & & & & & & & & \\
\hline 20 & 5308020501001 & 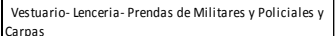 & 55376,64 & 55376,64 & & & & & & & & & \\
\hline 212 & $\begin{array}{l}5308030501001 \\
55008040501001\end{array}$ & 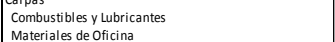 & $\begin{array}{l}29470,93 \\
321204.4\end{array}$ & $\begin{array}{l}29470,93 \\
3212044\end{array}$ & & & & & & & & & \\
\hline${ }_{23}$ & $\begin{array}{c}5080050501001 \\
01\end{array}$ & 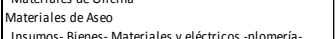 & 13477,13 & 13477,13 & & & & & & & & & \\
\hline 24 & 5308110501001 & 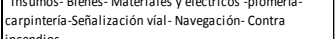 & 93449,97 & 93649,97 & & & & & & & & & \\
\hline
\end{tabular}

Una vez preparada la información en Excel, se diseñó la Aplicación Informática

SysCostos en la cual se ingresó la información y se obtuvo reportes de manera automática. 


\section{Estudio de costos invertidos en la formación universitaria de los estudiantes de la Facultad de Ciencias Administrativas de la Universidad Técnica de Cotopaxi}

Vol. 3, núm. 2., (2019)

Myrian del Rocío Hidalgo Achig; Marcela Patricia Vizuete Achig; Mayra Alexandra Chicaiza Herrera; Isabel Regina Armas Heredia; Edison Patricio Bedon Salazar; Patricia Geraldina López Fraga

\section{Cuadro 5. Reportes de la Aplicación Informática SysCostos}

\begin{tabular}{|c|c|c|c|c|c|c|}
\hline COSTOS INDIRECTOS & $\$$ & $1,066,529.76$ & & & & \\
\hline \multicolumn{2}{|l|}{ FACULTAD } & \multicolumn{5}{|c|}{ CIENCIAS ADMINISTRATIVAS } \\
\hline SERVICIOS & $\$$ & $93,138.77$ & $\$$ & $146,618.45$ & $\$$ & $54,280.87$ \\
\hline Item & \multicolumn{2}{|r|}{ COMERCIO } & \multicolumn{2}{|c|}{ CONTABILIDAD } & \multicolumn{2}{|c|}{ SECRETARIADO } \\
\hline Servicios Basicos & $\$$ & $10,502.48$ & $\$$ & $16,532.94$ & $\$$ & $6,120.80$ \\
\hline Servicios Generales & $\$$ & $7,560.11$ & $\$$ & $11,901.07$ & $\$$ & $4,406.00$ \\
\hline Arrendamiento de Bienes & $\$$ & 287.58 & $\$$ & 452.71 & $\$$ & 167.60 \\
\hline Contratacion de Estudios e Investigaciones & $\$$ & $13,325.12$ & $\$$ & $20,976.32$ & $\$$ & $7,765.82$ \\
\hline Gastos en Informatica & $\$$ & 724.31 & $\$$ & $1,140.20$ & $\$$ & 422.12 \\
\hline Bienes de Uso y Consumo Corriente & $\$$ & $12,928.81$ & $\$$ & $20,352.45$ & $\$$ & $7,534.85$ \\
\hline Gastos Financieros y Otros & $\$$ & $1,240.97$ & $\$$ & $1,953.53$ & $\$$ & 723.23 \\
\hline Gestion de la Investigacion & $\$$ & $46,569.39$ & $\$$ & $73,309.23$ & $\$$ & $27,140.44$ \\
\hline DEPRECIACIONES & $\$$ & $57,375.40$ & $\$$ & $90,319.98$ & $\$$ & $33,438.13$ \\
\hline Depreciacion Edificios & $\$$ & $21,881.99$ & $\$$ & $34,446.49$ & $\$$ & $12,752.73$ \\
\hline Depreciaciòn Vehiculos & $\$$ & 497.21 & $\$$ & 782.70 & $\$$ & 289.77 \\
\hline Depreciaciòn Maquinaria y Equipos & $\$$ & $13,019.76$ & $\$$ & $20,495.62$ & $\$$ & $7,587.86$ \\
\hline Depreciaciòn Equipos sistemas y paquetes informa & $\$$ & $15,861.37$ & $\$$ & $24,968.87$ & $\$$ & $9,243.94$ \\
\hline Depreciaciòn Mobiliarios & $\$$ & $6,115.06$ & $\$$ & $9,626.29$ & $\$$ & $3,563.83$ \\
\hline ADMINISTRATIVOS & $\$$ & $187,317.13$ & $\$$ & $294,873.41$ & $\$$ & $109,167.62$ \\
\hline COSTOS DIRECTOS & $\$$ & $1,393,477.58$ & & & & \\
\hline TIPOLOGIA & $\$$ & $488,236.03$ & $\$$ & $594,459.99$ & $\$$ & $310,781.55$ \\
\hline Item & & COMERCIO & & NTABILIDAD & & ETARIADO \\
\hline DOCENCIA & $\$$ & $325,542.30$ & $\$$ & $404,411.47$ & $\$$ & $217,065.41$ \\
\hline GESTION & $\$$ & $99,640.05$ & $\$$ & $89,418.94$ & $\$$ & $37,483.35$ \\
\hline INVESTIGACION & $\$$ & $32,230.35$ & $\$$ & $64,304.72$ & $\$$ & $40,217.56$ \\
\hline OTROS GASTOS & $\$$ & $19,069.84$ & $\$$ & $30,019.62$ & $\$$ & $11,113.82$ \\
\hline BECAS & $\$$ & $11,753.49$ & $\$$ & $6,305.24$ & $\$$ & 4,901.42 \\
\hline
\end{tabular}

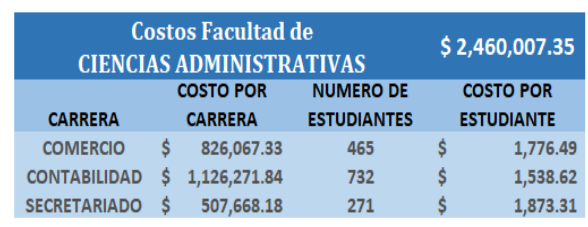

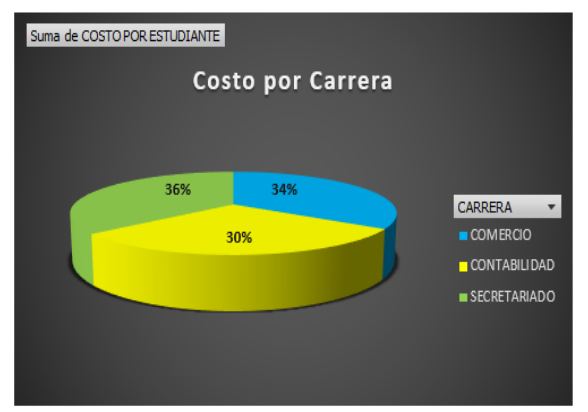

En cuanto a las FUENTES DE FINANCIAMIENTO de la Universidad Técnica de

Cotopaxi, la Ley Orgánica de Educación Superior en el Titulo II Capitulo II, correspondientes a la constitución y financiamiento de las instituciones de educación superior señala lo siguiente:

a) Los bienes muebles e inmuebles que al promulgarse esta Ley sean de su propiedad, y los bienes que se adquieran en el futuro a cualquier título, así como aquellos que fueron ofertados y comprometidos al momento de presentar su proyecto de creación;

b) Las rentas establecidas en la Ley del Fondo Permanente de Desarrollo Universitario y Politécnico (FOPEDEUPO); 


\section{Estudio de costos invertidos en la formación universitaria de los estudiantes de la Facultad de Ciencias Administrativas de la Universidad Técnica de \\ Cotopaxi}

Vol. 3, núm. 2., (2019)

Myrian del Rocío Hidalgo Achig; Marcela Patricia Vizuete Achig; Mayra Alexandra Chicaiza Herrera; Isabel Regina Armas Heredia; Edison Patricio Bedon Salazar; Patricia Geraldina López

Fraga

c) Las asignaciones que han constado y las que consten en el Presupuesto General del Estado, con los incrementos que manda la Constitución de la República del Ecuador;

d) Las asignaciones que corresponden a la gratuidad para las instituciones públicas;

e) Los ingresos por matrículas, derechos y aranceles, con las excepciones establecidas en la Constitución y en esta Ley en las universidades y escuelas politécnicas públicas;

f) Los beneficios obtenidos por su participación en actividades productivas de bienes y servicios, siempre y cuando esa participación no persiga fines de lucro y que sea en beneficio de la institución;

g) Los recursos provenientes de herencias, legados y donaciones a su favor;

h) Los fondos autogenerados por cursos, seminarios extracurriculares, programas de posgrado, consultorías, prestación de servicios y similares, en el marco de lo establecido en esta Ley;

i) Los ingresos provenientes de la propiedad intelectual como fruto de sus investigaciones y otras actividades académicas;

j) Los saldos presupuestarios comprometidos para inversión en desarrollo de ciencia y tecnología y proyectos académicos y de investigación que se encuentren en ejecución no devengados a la finalización del ejercicio económico, obligatoriamente se incorporarán al presupuesto del ejercicio fiscal siguiente; 
Estudio de costos invertidos en la formación universitaria de los estudiantes de la Facultad de Ciencias Administrativas de la Universidad Técnica de Cotopaxi

Vol. 3, núm. 2., (2019)

Myrian del Rocío Hidalgo Achig; Marcela Patricia Vizuete Achig; Mayra Alexandra Chicaiza Herrera; Isabel Regina Armas Heredia; Edison Patricio Bedon Salazar; Patricia Geraldina López Fraga

k) Los recursos obtenidos por contribuciones de la cooperación internacional; y,

1) Otros bienes y fondos económicos que les correspondan o que adquieran de acuerdo con la Ley.

Figura 2. Ingresos por Naturaleza
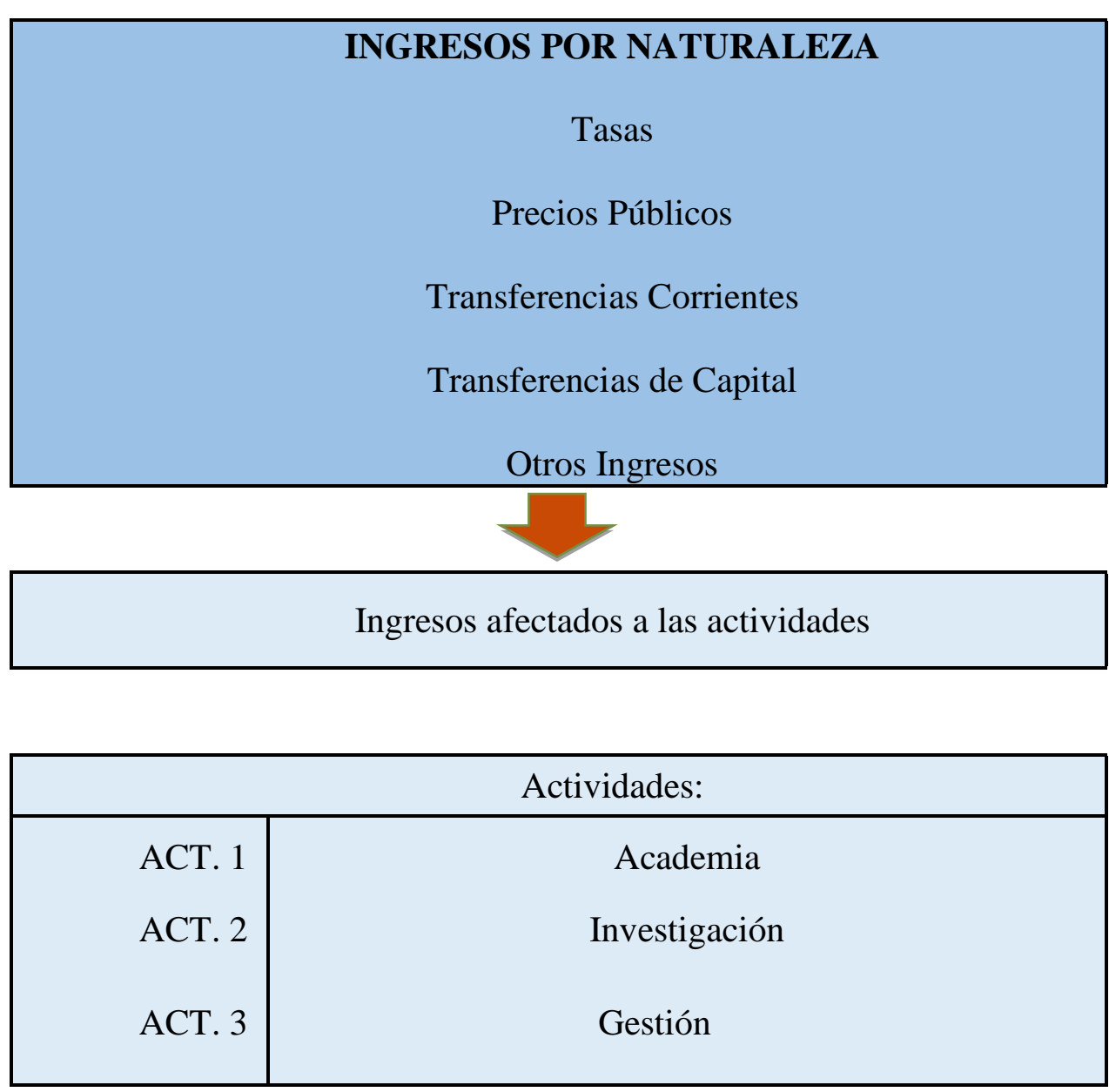

Figura 2. Clasificación Funcional de los Ingresos

Elaborado por: Grupo de Investigación 


\section{Estudio de costos invertidos en la formación universitaria de los estudiantes de la Facultad de Ciencias Administrativas de la Universidad Técnica de Cotopaxi}

Vol. 3, núm. 2., (2019)

Myrian del Rocío Hidalgo Achig; Marcela Patricia Vizuete Achig; Mayra Alexandra Chicaiza Herrera; Isabel Regina Armas Heredia; Edison Patricio Bedon Salazar; Patricia Geraldina López

Fraga

Ingresos que afectan a las actividades

Los ingresos que afectan a las actividades se les han designado de acuerdo al siguiente cuadro en donde se especifica cada una de las actividades

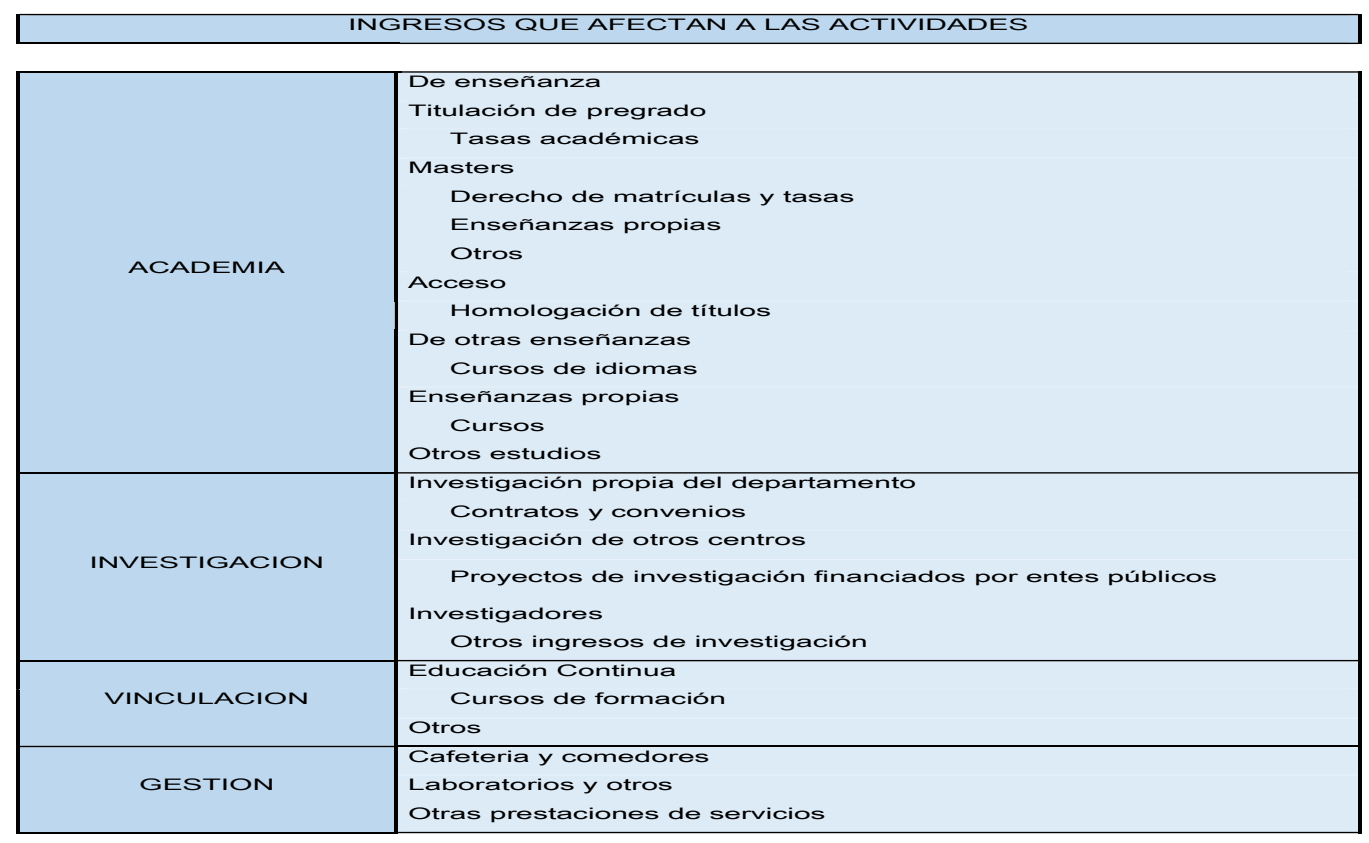

Figura 3. Ingresos que Afectan A Actividades

\section{Resultados.}

Una vez desarrollada la metodología descrita anteriormente, se obtuvo los siguientes resultados en la Facultadad de Ciencias Administrativas:

- Los Costos por Facultad ascendieron a \$2`460.007,35 durante el año 2017

- Los costos incurridos por carreras fueron: 


\section{Estudio de costos invertidos en la formación universitaria de los estudiantes de la Facultad de Ciencias Administrativas de la Universidad Técnica de Cotopaxi}

Vol. 3, núm. 2., (2019)

Myrian del Rocío Hidalgo Achig; Marcela Patricia Vizuete Achig; Mayra Alexandra Chicaiza Herrera; Isabel Regina Armas Heredia; Edison Patricio Bedon Salazar; Patricia Geraldina López Fraga
Comercio:
\$ $826.067,33$
Contabilidad:
$\$ 11^{\prime} 126.271,84$
Secretariado:
$\$ 507.668,18$

- Finalmente, los costos por estudiante ascendieron:

$\begin{array}{lll}\text { Comercio: } & & \$ 1776,49 \\ & & \\ \text { Contabilidad: } & & \$ 1538,62 \\ & & \\ \text { Secretariado: } & & \$ 1873,31\end{array}$

Con esta información se puede mencionar que la carrera que genera mayores costos es la de Contabilidad, mientras que la carrera que genera menores costos es la carrera de Secretariado ejecutivo esto se debe al número de estudiantes matriculados que cada carrera manejo durante el año 2017.

\section{Conclusiones.}

- El sistema de Contabilidad Gubernamental Esigef registra la ejecución presupuestaria de la Universidad, en virtud que su estructura obedece a lineamientos definidos para el control presupuestario - contable de las entidades públicas del país. Por tanto, no se ha definido un mecanismo que permita reflejar los costos de las carreras de las universidades. 


\section{Estudio de costos invertidos en la formación universitaria de los estudiantes de la Facultad de Ciencias Administrativas de la Universidad Técnica de \\ Cotopaxi}

Vol. 3, núm. 2., (2019)

Myrian del Rocío Hidalgo Achig; Marcela Patricia Vizuete Achig; Mayra Alexandra Chicaiza Herrera; Isabel Regina Armas Heredia; Edison Patricio Bedon Salazar; Patricia Geraldina López

Fraga

- La Facultad de Ciencias Administrativas de la Universidad Técnica de Cotopaxi no cuenta con un sistema que le permita el manejo y control de costos de sus facultades, extensiones, centros y carreras.

- La estructura financiera de la facultad denota autonomía de la Administración de la Universidad, sin embargo, su presupuesto no permite alcanzar la importancia necesaria para obtener un avance significativo en el desarrollo de las carreras de la Facultad.

- La facultad obtiene sus recursos económicos de la proforma presupuestaria de la Universidad, sin considerar un análisis de costos por carreras lo que provoca que sus requerimientos los efectué en base a necesidades urgentes y permanentes, lo que refleja un escaso desarrollo en su infraestructura académica y tecnológico de la Facultad.

- La facultad no se encuentra obligada a presentar los costos por carrera, sin embargo, se hace menester contar con un instrumento de medición y control en cuantos a costos e ingresos.

- Los costos por carreras y Facultad se han determinado con el diseño de una aplicación informática denominada "SysCostos" en la que se procedió a ingresar información generada en matrices correspondiente al año 2017, por cada uno de los grupos de trabajo.

- Los Costos se procesaron en base a determinación de los costos directos e indirectos y para estos últimos se determinaron bases de asignación para su distribución. 
Estudio de costos invertidos en la formación universitaria de los estudiantes de la Facultad de Ciencias Administrativas de la Universidad Técnica de Cotopaxi

Vol. 3, núm. 2., (2019)

Myrian del Rocío Hidalgo Achig; Marcela Patricia Vizuete Achig; Mayra Alexandra Chicaiza Herrera; Isabel Regina Armas Heredia; Edison Patricio Bedon Salazar; Patricia Geraldina López Fraga

\section{Bibliografía.}

Bravo Valdivieso, M. (2007). Contabilidad de Costos. Quito: Nuevodía.

Goncalves, L., Do Nascimento, C., Dos Santos, M., \& Bernardes, S. (7 de 2013). Sistemas de Costes: Importancia, Viabilidad y Utilidad en la Concepción de los Agentes Públicos del Estado de Paraná (Brasil). Revista de Educação e Pesquisa em Contabilidade, 7(2), 168 184.

Macias, M. (4 de 04 de 2011). Advenio . Obtenido de https://advenio.es/la-estructura-de-costesde-tu-empresa/

Martinez Navajas, J. (2006). Contabilidad Analítica (Primera ed.). Escuela de Negocios eoi. $\begin{array}{lllllll}\text { Recuperado el } & 2017 & \text { de } & 07 & \text { de } & 1, & \text { de }\end{array}$ https://static.eoi.es/savia/documents/componente45061.pdf

Zapata, J. (2007). Contabilidad de Costos. México: Mcgraw Hill. 